\title{
Investigation of the First Spermatorrhea Age of Males in Jiangsu Province, China
}

\author{
Fang Lyu1,2,3*, Lin Liu ${ }^{1 *}$, Xin Wang ${ }^{1}$, Yadong $\mathrm{Li}^{4}$, Yurong Ji1,2, Tonghui Meng1, Zhe Zhang5, \\ Ning Wang5\#, Shucheng Zhang5\# \\ ${ }^{1}$ Department of Obstetrics and Gynecology, Yangzhou University, Yangzhou, China \\ ${ }^{2}$ Reproductive Medicine Center, Department of Obstetrics and Gynecology, Clinical Medical College, Yangzhou University, \\ Northern Jiangsu People's Hospital, Yangzhou, China \\ ${ }^{3}$ Department of Obstetrics and Gynecology, Third Hospital, Peking University, Beijing, China \\ ${ }^{4}$ Obstetrics and Gynecology Hospital of Fudan University, Shanghai, China \\ ${ }^{5}$ Department of Cell Biology, National Research Institute of Family Planning, Beijing, China \\ Email: "wangn333@126.com, "nrifp@263.net
}

How to cite this paper: Lyu, F., Liu, L., Wang, X., Li, Y.D., Ji, Y.R., Meng, T.H., Zhang, Z., Wang, N. and Zhang, S.C. (2020) Investigation of the First Spermatorrhea Age of Males in Jiangsu Province, China. Health, 12, 523-531

https://doi.org/10.4236/health.2020.126041

Received: April 20, 2020

Accepted: May 29, 2020

Published: June 2, 2020

Copyright (c) 2020 by author(s) and Scientific Research Publishing Inc. This work is licensed under the Creative Commons Attribution International License (CC BY 4.0)

http://creativecommons.org/licenses/by/4.0/

(c) (i) Open Access

\begin{abstract}
Background: The growth of adolescents is affected by economic development and education levels. We investigated the first spermatorrhea age of adolescents in urban area and in rural area of Jiangsu province, China. Methods: The data were obtained through anonymous questionnaire by using cross-sectional methods. From May 2016 to March 2017 in Jiangsu province, China, a total of 2131 Han nationality students were enrolled in cluster. The survey was contained date of birth, age, grade, age of first spermatorrhea and how long ago of the first spermatorrhea, all data were analyzed by Prism 8.0. Results: The questionnaire accounted for response was $97.3 \%$, and the effective rate was $96.3 \%$. The mean age of first spermatorrhea was (13.7 \pm 1.5$)$ years old, which included $13.6 \pm 1.4$ years in urban and $13.9 \pm 1.5$ years in rural. The first spermatorrhea occurred in the fifth and sixth grade primary school, and 1,2, 3 grades of junior high school were $3.5 \%, 13 \%, 27.4 \%, 28.5 \%$ and $15.1 \%$, respectively. There was $62.3 \%$ of first spermatorrhea occurred in summer. The rate of spermatorrhea with natural occurrence accounted for $95.1 \%$, and with active accounted for $4.9 \%$. Conclusions: Compared with rural area, the age of the first spermatorrhea of males in urban area is significant earlier. The adolescent's first spermatozoa were mostly occurred in summer. The relevant physical and reproductive education should be started from the third grade of primary school. The reproductive health education should be carried out when the youth are 7 years old.
\end{abstract}

\section{Keywords}

Male, Adolescents, First Spermatozoa, Puberty Development, Jiangsu, China

${ }^{\star} \mathrm{FL}$ and LL contributed equally to the work. 


\section{Introduction}

The important period for children to adults is adolescences [1]. During the period of adolescence, skeletal muscles grow rapidly, brain neuron structure and the function are initially perfected, and the fecundity is gradually matured [2]. Puberty development is influenced by individual genes, environmental factors, and economic and social levels. The level of maturity is evident in different countries, regions, races, and individuals. Even in the same region, different economic and cultural have different ages of maturity [3]. The growth and mental health of adolescents are affected by economic development and education levels [4]. Qiu investigated 3786 male college students in Jiangsu showed that $68.78 \%$ experienced their first spermatorrhea at the age of 12 - 15 [5]. Li surveyed 367 boys aged 8 - 15 years found that the average age at the first spermatorrhea was 12.15 years, and prenatal androgen exposure may relate to the first spermatorrhea [6]. In women, menarche is an obvious sign when puberty is launched. In men, a sign of maturity in male puberty is semen urine or acquired premature ejaculation. Compared with women, men's nocturnal emission has the hallmark of menstrual menarche. However, the discovery and determination of the first spermatorrhea were certain inaccuracy. Hence, our project compared the adolescents first spermatorrhea age in urban area and in rural area of Jiangsu, China.

\section{Method}

\subsection{Survey Object and Defining Sample Size}

From May 2016 to March 2017, the survey included males from 1 primary school, 3 middle schools and 3 junior high schools in Jiangsu, China. Our project is planning two independent means of basic physiological parameters of adolescences which is continuous data, to investigate the first spermatorrhea age by a predetermined age of $15(\delta=15)$. For a two-tail significance test, with $80 \%$ power and $5 \%$ level of significance, $z_{\alpha}$ value is 1.96 and $z_{\beta}=0.842$ [7]. Assume that the data sampled are normally distributed, equal variance for both compared groups and equal numbers in each group. The sample size is 142 in each group showed in Figure 1. The study was approved by the institutional ethics review committee of Northern Jiangsu People's Hospital and the consents were obtained from study participants was written.

\subsection{Data Acquisition}

\subsubsection{Cross-Sectional Stratified Cluster Methods}

The paper and electronic questionnaire were unified by using national science

$$
\begin{aligned}
& \mathrm{n}=2\left[\frac{\left(Z_{\alpha}+Z_{\beta}\right) \delta}{E S}\right]^{2} \\
& \mathrm{n}=2\left[\frac{(1.96+0.84) 15}{5}\right]^{2}=142 \text { in each group }
\end{aligned}
$$

Figure 1. Sample size formula for the first spermatorrhea age means. 
and technology basic work project. The questionnaire was examined and approved by the consulting experts and the guiding group. Standard operation procedure (SOP) included the training of investigators, investigation process and post-investigation data processing. All of the information was registered in information management platform system and recorded on files. The anonymous questionnaire was randomly filled out number.

\subsubsection{Questionnaire Filling}

The questionnaire was included the date of birth, years of survey, age, grade, first spermatorrhea (years, months) and how long ago the first spermatorrhea occurred. There were a total of nine indicators to investigate the puberty development, such as grade, age, distribution, season and state of occurrence in the first spermatorrhea. The age of the first spermatorrhea was accurate in the month and quarter by self-reported participants. Adolescents with normal physical exercise activities, development and good health were included, but excluded non-Han nation males, blanked of spermatorrhea information, information with special disease were obvious influence growth and development, refusal questionnaires with logical errors or inaccurate.

\subsection{Control Variables}

The obtained questionnaire was verified in parallel and data screening through logical judgment by two researchers, clarified and submitted to two investigators. When the data is heterogeneity, the third researcher was needed to participate into the discussion to clarify. The data was entered by the third researcher checked out and determined the logical verification information. The analysis should be consistent with accurately and logically determined information.

\subsection{Statistical Analysis}

According to the area of life, the survey data was obtained to meet the requirements by dividing into in urban area group and in rural area group. But when the household registration did not match the residence, we chose the main living place of residence grouping by principle. The unit of the age of the first spermatorrhea was month. Average mid-month calculation, survey accuracy is 1 month (error control in \pm 1 month). All data were used by Prism 8.0 for ANOVA and T-TEST.

\section{Results}

\subsection{Survey Situation}

A total of 2335 questionnaires was sent out, and 2274 questionnaires were collected, the recovery rate was $97.4 \%$. The effective data questionnaire was 2192 , included 2131 Han nationality and 61 other nationalities, the effective questionnaire rate was $96.4 \%$.

\subsection{Survey Object General Situation}

Analysis of 2131 survey success questionnaires, among the respondents Han 
male height, weight, body mass index (BMI) urban area are higher than rural area $(\mathrm{P}<0.05)$. The basic physiological indicators are shown in Table 1.

\subsection{Age of First Spermatorrhea}

The data showed that the first time the male spermatorrhea is $(13.7 \pm 1.4)$ years old; $13.6 \pm 1.4$ years in urban and $13.9 \pm 1.5$ years in rural. Compared the age of first spermatorrhea, urban is earlier than rural, $\mathrm{P}=0.01$, Table 2 .

\subsection{Distribution of Age at the Time of First Spermatorrhea}

The earliest first spermatorrhea is 7.8 years old and 18.3 years old is the oldest. The time of first spermatorrhea occurred in the 13 and 14 years old, which the urban is $28.4 \%$ and $28.8 \%$, rural is $23.0 \%$ and $27.2 \%$, respectively, Table 3 and Table 4 .

\subsection{Seasonal Distribution of the First Spermatorrhea}

The occurrence of the first nocturnal emission was counted in spring, summer, autumn and winter. Both urban and rural males are highest frequency of first spermatorrhea in summer ( $>50 \%)$, but winter was the least $(<10 \%)$, Table 5.

\subsection{Emission Nocturnal Occurs in All Grades}

The age of the first spermatorrhea showed that the second and the third the grades in middle school had reached $73.8 \%$ and $89.1 \%$ (Table 6), which was consistent with Table 3 and Table 4.

Table 1. Basic physiological parameters.

\begin{tabular}{cccc}
\hline & Urban area & Rural area & P value \\
\hline Number of cases & 1589 & 542 & \\
Age & $16.5 \pm 1.3$ & $14.4 \pm 2.8$ & $<0.00^{\star *}$ \\
Height $(\mathrm{cm})$ & $173.4 \pm 7.2$ & $164.7 \pm 13.2$ & $<0.00^{* *}$ \\
Weight $(\mathrm{kg})$ & $64.0 \pm 12.3$ & $57.2 \pm 16.7$ & $<0.00^{* *}$ \\
BMI $\left(\mathrm{kg} / \mathrm{m}^{2}\right)$ & $21.3 \pm 3.7$ & $20.7 \pm 4.2$ & $<0.00^{* *}$ \\
\hline
\end{tabular}

All were significant at $\mathrm{p}<0.001$ compared between urban and rural area, ${ }^{* *}$ significant at $\mathrm{P}<0.00$.

Table 2. Distribution of the age of first spermatorrhea in household registration.

\begin{tabular}{cccc}
\hline & Urban area & Rural area & P value \\
\hline Cases & 1589 & 542 & \\
Age (months) & $173.4 \pm 7.2$ & $164.7 \pm 13.2$ & 0.70 \\
Age (years) & $13.6 \pm 1.4$ & $13.9 \pm 1.5$ & $0.01^{*}$ \\
\hline
\end{tabular}

*significant at $\mathrm{P}<0.01$ compared between urban and rural.

Table 3. The age of first spermatorrhea of distribution (\%).

\begin{tabular}{cccccccccccccc}
\hline & Case & 7 & 8 & 9 & 10 & 11 & 12 & 13 & 14 & 15 & 16 & 17 & 18 \\
\hline Urban & 1170 & 0.1 & 0.2 & 0.2 & 1.5 & 3.7 & 12.7 & 28.4 & 28.8 & 14.4 & 7.7 & 2.2 & 0.1 \\
Rural & 265 & 0.0 & 0.0 & 0.8 & 0 & 2.6 & 14.0 & 23.0 & 27.2 & 18.1 & 9.8 & 4.5 & 0.0 \\
Total & 1435 & 0.1 & 0.1 & 0.3 & 1.3 & 3.5 & 13.0 & 27.4 & 28.5 & 15.1 & 8.1 & 2.6 & 0.1 \\
\hline
\end{tabular}


Table 4. Cumulative of the age of first spermatorrhea (\%).

\begin{tabular}{cccccccccccccc}
\hline & Case & 7 & 8 & 9 & 10 & 11 & 12 & 13 & 14 & 15 & 16 & 17 & 18 \\
\hline Urban & 1170 & 0.1 & 0.3 & 0.4 & 2.0 & 5.6 & 18.4 & 46.8 & 75.6 & 90.0 & 97.7 & 99.9 & 100 \\
Rural & 265 & 0.0 & 0.0 & 0.8 & 0.8 & 3.4 & 17.4 & 40.4 & 67.5 & 85.7 & 95.5 & 100 & 100 \\
Total & 1435 & 0.1 & 0.2 & 0.5 & 1.8 & 5.3 & 18.4 & 45.1 & 73.8 & 89.1 & 97.3 & 99.9 & 100 \\
\hline
\end{tabular}

Table 5. The first spermatorrhea distribution of seasons (case \%).

\begin{tabular}{cccccc}
\hline & Case & Spring & Summer & Autumn & Winter \\
\hline Urban & 1170 & $297(25.4)$ & $600(51.3)$ & $206(17.6)$ & $67(5.7)$ \\
Rural & 265 & $42(15.8)$ & $169(63.7)$ & $31(11.6)$ & $23(8.9)$ \\
Total & 1435 & $339(23.6)$ & $769(53.6)$ & $237(16.5)$ & $90(6.3)$ \\
\hline
\end{tabular}

Table 6. The first spermatorrhea of grade distribution.

\begin{tabular}{ccccccccccccc}
\hline & \multicolumn{4}{c}{ Elementary school } & \multicolumn{3}{c}{ Middle school } & \multicolumn{3}{c}{ High school } \\
\hline & Case & 2 & 3 & 4 & 5 & 6 & 1 & 2 & 3 & 1 & 2 & 3 \\
\hline Urban & 1170 & 0.4 & 0.8 & 1.6 & 6.2 & 7.8 & 26.7 & 36.7 & 18.2 & 1.4 & 0.1 & 0 \\
Rural & 265 & 0 & 2.0 & 8.4 & 17.7 & 10.8 & 14.5 & 28.9 & 14.9 & 2.8 & 0 & 0 \\
Total & 1435 & 0.4 & 1.0 & 2.9 & 8.3 & 8.3 & 24.4 & 35.3 & 17.6 & 1.6 & 0.1 & 0 \\
\hline
\end{tabular}

\subsection{State at the Time of the First Spermatorrhea}

1435 out of 2131 males showed the status of the first spermatorrhea occurred. There were 1365 with nighttime sleep (natural spermatorrhea) (95.1\%), 13 males with natural spermatorrhea (0.9\%), and 46 males with masturbating behavior $(3.2 \%)$. Simultaneously, with regarding to sexual activity, when the first spermatorrhea, 5 males $(0.4 \%)$ with others (friends, classmates) and 6 males in sexual activity $(0.4 \%)$.

\section{Discussion}

Adolescent development is not only an important period in the adolescent stage, but also has physical health, mental health and self-awareness in adulthood important influence of life [8]. Basic physiological parameters were related to adolescent development, including the development of adolescent health education and service strategies providing support [9]. There are less studies of male puberty and the age of first spermatorrhea in China. But existing reports are in survey design, data collection and the quality control of methodologies had limited description, or a methodological bias in the accuracy of the conclusion [5] [10] [11]. Therefore, it is imperative to establish standards of data collection and specifications of implementation during the investigation.

Our research is the first time reported the first occurrence of nocturnal emission in urban area and rural area of Jiangsu province. The data showed that the mean age of the first spermatorrhea was 13.7 years old, and area the first sper- 
matorrhea age in urban is significant earlier than in rural area. The age of the first spermatorrhea had reached $89.1 \%$ in the third year of middle school, and mostly occurred in summer. The earliest first spermatorrhea is 7.8 years old. The most of nocturnal emission has emerged from wet dreams, and $4.9 \%$ of the total males with active situation including sexual related activity. To guarantee the comparability of data collected from different regions and different ages, the data was carried out in accordance with the uniform requirements of the national investigations and became part of national physiological database [9] [12].

Males have a quick physical development of brain function, spirit, behavior, and physiologic function in puberty, but their reproduction knowledge is not yet fully matched their development [13]. Regardless of the number or individual height development peak, the first spermatorrhea of male puberty development was major in the 13 - 15 years old in each group [2]. Zhang found that spermatorrhea was significantly decreased in the observation males, but there was no significant difference in the age of the first spermatorrhea [14]. Health education, self-enhancement should be in the sixth grade, to adolescent health education protection, contraception, sexually transmitted diseases [6] [15]. Su reported that early menarche adolescent girls positively relates to bullying victimization and perpetration [16], which suggested early puberty predicts involvement in school bullying. It calls for joint attention from schools, families and the community to establish special research and education on sexual health. The content of reproductive health education for male adolescent should be included contraception. A limitation was that male physiological parameters in our study were based on self-reported data from the participants; longitudinal studies are needed to clarify the relationship between basic physiological parameters and the first spermatorrhea age in urban area and in rural area.

\section{Conclusion}

In conclusion, the mean first spermatorrhea age was 13.6 years in urban and 13.9 years in rural, and the age of the first spermatorrhea of males in urban area is significant earlier than rural area. The first spermatorrhea age had reached $89.1 \%$ in the third years in middle school, which mostly occurred in summer. $0.4 \%$ males had the first spermatorrhea with sexual activity, and the earliest first spermatorrhea is 7.8 years old. Therefore, their sexual health calls for schools provide health education services which are compatible with age and physical development to promote the healthy, scientific and sustainable development of adolescents.

\section{Ethics Approval and Consent to Participate}

The study was approved by the institutional ethics review committee of Northern Jiangsu People's Hospital.

\section{Consent for Publication}

All data generated during this study were obtained from study participants that 
were written for publication.

\section{Availability of Data and Material}

The data set analyzed is available from the corresponding author on reasonable request.

\section{Funding}

This study was supported by using National Major Science and Technology Projects of China (No. 2013FY110500), the National Natural Science Foundation of China (No.81601343), six talent peaks project (No. WSW-197) and talent medical youth in Jiangsu Province (No.QNRC2016345).

\section{Authors' Contributions}

FL and SCZ conducted the data analyses and manuscript writing; FL and NW designed the study and the outline of the manuscript; SCZ, FL and YDL designed the questionnaire, LL, XW, YRJ, THM and ZZ collected the data. All authors read and approved the final manuscript.

\section{Acknowledgements}

We would like to acknowledge all the participants and the university faculties for participating in this study.

\section{Conflicts of Interest}

The authors declare that they have no competing interests.

\section{References}

[1] Sawyer, S.M., Azzopardi, P.S., Wickremarathne, D. and Patton, G.C. (2018) The Age of Adolescence. The Lancet Child \& Adolescent Health, 2, 223-228. https://doi.org/10.1016/S2352-4642(18)30022-1

[2] Patton, G.C. and Viner, R. (2007) Pubertal Transitions in Health. Lancet, 369, 1130-1139. https://doi.org/10.1016/S0140-6736(07)60366-3

[3] Viner, R.M., Ozer, E.M., Denny, S., Marmot, M., Resnick, M., Fatusi, A. and Currie, C. (2012) Adolescence and the Social Determinants of Health. Lancet (London, England), 379, 1641-1652. https://doi.org/10.1016/S0140-6736(12)60149-4

[4] Patton, G.C., Sawyer, S.M., Santelli, J.S., Ross, D.A., Afifi, R., Allen, N.B., et al. (2016) Our Future: A Lancet Commission on Adolescent Health and Wellbeing. Lancet (London, England), 387, 2423-2478. https://doi.org/10.1016/S0140-6736(16)00579-1

[5] Qiu, D.X., Wang, H., Luo, Y.H., Pang, X.M., Zhang, Y.W., Shi, J.H., Li, Y.G., Lin, Y. and Liu, J. (2008) Sexual Physiology and Psychology of Male College Students and Their Clinical Significance. Zhonghua Nan Ke Xue, 14, 903-906.

[6] Li, T., Wu, L., Wang, Y., Yao, R., Meng, Y., Zhang, Y., Li, Z., Zhou, Y., Han, H. and Fu, L. (2018) The Association between Digit Ratio (2d:4d) and the First Spermatorrhea among Chinese Boys. Early Human Development, 118, 48-52. https://doi.org/10.1016/j.earlhumdev.2018.01.016 
[7] Malone, H.E., Nicholl, H. and Coyne, I. (2016) Fundamentals of Estimating Sample Size. Nurse Researcher, 23, 21-25. https://doi.org/10.7748/nr.23.5.21.s5

[8] Sawyer, S.M., Afifi, R.A., Bearinger, L.H., Blakemore, S.J., Dick, B., Ezeh, A.C. and Patton, G.C. (2012) Adolescence: A Foundation for Future Health. Lancet (London, England), 379, 1630-1640. https://doi.org/10.1016/S0140-6736(12)60072-5

[9] Zhang, S., He, B. and Ren, T. (2005) Evaluation of Male Semen Quality in China and the Significance of Establishing a Database of Reproductive Resources. Chinese Journal of Family Planning, 15, 468-470.

[10] Zhou, X., Wang, N. and Zhang, S. (2016) Investigate the Age of the First Spermatozoa of Chinese Adolescents in 2014. Chinese Journal of Clinicians, 10, 3228-3233.

[11] Li, Y., Lyu, F., Hu, N., Bao, J., Bao, Y., Chen, B., Wang, N., Zhang, S. and Gu, Y. (2018) The Survey of Mongolian Adolescents in the Age of the Spermatorrhea. Chinese Journal of Family Planning, 26, 357-361.

[12] Zhang, S., He, B., Cheng, J. and Wang, J. (2012) The Trend of Male Reproductive Health and Semen Quality in China. China Science and Technology Achievements, 13, 69-71.

[13] Patton, G.C., Olsson, C.A., Skirbekk, V., Saffery, R., Wlodek, M.E., Azzopardi, P.S., et al. (2018) Publisher Correction: Adolescence and the Next Generation. Nature, 559, E1. https://doi.org/10.1038/s41586-018-0069-3

[14] Zhang, L.D., Li, H.C., Gao, M., Wang, L., Deng, Q., Shi, T., He, J.L., Chong, T. and Wang, Z.M. (2013) Sexual Development Characteristics and Sex Hormone Levels in Obese Male Adolescents. Zhonghua Nan Ke Xue, 19, 434-438.

[15] Gao, Y., Zhang, S. and He, B. (2009) The Age of the First Male Spermatozoa in China. Chinese Journal of Family Planning, 17, 653-658.

[16] Su, Q., Chen, Z., Li, R., Elgar, F.J., Liu, Z. and Lian, Q. (2018) Association between Early Menarche and School Bullying. Journal of Adolescent Health, 63, 213-218. https://doi.org/10.1016/j.jadohealth.2018.02.008 


\section{Abbreviations}

SOP: Standard operation procedure; BMI: Body Mass Index.

\section{Survey of Chinese Male Physiological and the First Spermatorrhea Age}

This survey is an integral part of the basic data on the development of national scientific strategy. Please be sure to complete it truthfully (required for each space). Thank for your cooperation.

1) Date of birth Year Month Age Nationality

2) Grade High school grade; Junior high school grade; Primary school grade

3) Father's Nationality _ Mother's Nationality

4) Height $\mathrm{cm}$, Weight $\mathrm{Kg}$,

5) Household registration: $1=$ Urban; $2=$ Rural; Residence: $1=$ Urban; $2=$ Rural

6) If you haven't spermatorrhea, you do not need to fill the items in the below (please tick $\sqrt{ }$ )

Note: The age below is the legal age $=$ actual age (please convert the age according to the virtual age of local custom)

7) The first spermatorrhea: Age _, how many years ago __ (please carefully consider your age of the first spermatorrhea)

8) The season is: (please tick $\sqrt{ }$ ) (1) Spring (2) Summer (3) Autumn (4) Winter (If it is not clear, it can be inferred according to the weather was cold or hot).

9) When it happened, what grade was it: (Please select, mark $O$ )

Primary school (grade) 2345 6; Junior high school (grade) 12 3; High school (grade) 123

10) Your first sperm discharge/spermatorrhea occurred in which of the following situations? (Single choice) (Please select and tick $\sqrt{ }$ )

1 = Sleeping at night: natural spermatorrhea (during non-wake sleep)

2 = Nap: natural nocturnal emission (during non-wake sleep)

3 = In awake state: self-active behavior (such as masturbation/masturbation, stimulation/rubbing, etc.)

$4=$ When with friends, classmates, partners, others (playing, touching, intimacy, etc.)

$5=$ When had sexual activity (during sexual activity with a clear purpose) 